\section{OP0101 COMPARISON OF SELECTED DISEASE ACTIVITY MARKERS IN SYSTEMIC LUPUS ERYTHEMATOSUS}

${ }^{1} \mathrm{P}$ Horák, ${ }^{1} \mathrm{~V}$ Ščudla, ${ }^{2} \mathrm{Z}$ Heřmanová, ${ }^{1} \mathrm{~L}$ Faltýnek, ${ }^{1} \mathrm{Z}$ Pospišil, ${ }^{3} \mathrm{M}$ Budíková. ${ }^{1} / l$ Ird Department of Internal Medicine; ${ }^{2}$ Department of Clinical Immunology; ${ }^{3}$ Department of Nuclear Medicine, University Hospital of Olomouc, Olomouc, Czech Republic

\subsection{6/annrheumdis-2001.185}

Background Systemic lupus erythematosus (SLE) is an autoimmune disorder, which affects multiple organ systems and which aetiology still remains unknown. The newer markers of the disease activity based on the phenomenon of autoantibody formation (anti-nucleosome antibodies), endothelial cell activation or damage (sVCAM-1, sICAM-1, thrombomodulin), activation of T or B cells (the variety of cytokines or their receptors), macrophage activation (neopterin), apoptosis (fas ligand, bcl-2 expression), complement impairment (C1q, complement split products) are lacking a widely accepted consensus on their utility.

Objectives The objectives of this study was to assess the utility of measurement of thrombomodulin, anti-nucleosome antibodies, sVCAM-1, sICAM-1, neopterin, fas ligand, IL-10, sIL-2R in patients with SLE and to compare them to traditional markers of SLE activity (anti-dsDNA antibodies, C3, C4), ECLAM index of disease activity and to each other.

Methods The measurement was done during six months period in three consecutive time points in each proband of the 52 patients with SLE. Anti-dsDNA antibodies, thrombomodulin, anti-nucleosome antibodies, sVCAM-1, sICAM-1, neopterin, fas ligand, IL-10, sIL-2R were tested by ELISA methods, C3, C4 components of complement by nefelometry. Pearson correlation test and ANOVA test were used for statistical evaluation of results.

Results Fas ligand and IL-10 do not correlate significantly with ECLAM index $(p>0.05)$. The rest of markers shows significant correlation with the disease activity index $(\mathrm{p}<0.05)$. Differences in plasma levels and fluctuation depending on the changes in disease activity index among the subgroups with stable, rising and dicreasing disease activity index are significant in the case of thrombomodulin and anti-dsDNA antibodies (ANOVA $\mathrm{p}<$ 0.05). Solubile VCAM-1, s-ICAM-1, sIL-2R, neopterin correlate with ECLAM index ( $\mathrm{p}<0.05)$, but their levels do not differ significantly between the subgroups (ANOVA $p>0.05$ ). Levels of anti-nucleosome antibodies correlate with ECLAM index and they are positive also in 21 sera with negative anti-dsDNA titer, but their levels do not differ significantly between the subgroups (ANOVA $\mathrm{p}>0.05$ ).

Conclusion Thrombomodulin and anti-dsDNA antibodies reflect in the best way the changing trend in disease activity. Anti-nucleosome antibody seems to be a promising a marker useful in early diagnosis. Solubile VCAM-1, sICAM-1, neopterin and sIL-2R are interesting molecules playing role in the disease pathogenesis but their practical utility stayes limited.

The financial support by IGA ÈR NK 4616-3 OK 10

\section{OP0102 INFECTION IN SYSTEMIC LUPUS ERYTHEMATOSUS (SLE), A 21 YEARS STUDY}

M Akbarian, S Shahnia, S Ehsani, F Gharibdoost, F Shahram, A Nadji, AR Jamshidi, F Davatchi. Reumatology Research Center, Shariati Hospital, Tehran, Iran

10.1136/annrheumdis-2001.186
Background Infection is a major source of morbidity and mortality in patients with SLE. The prevalence of infection in SLE is different in various countries and is due to many factors.

Objectives The aim of our study was to evaluate the incidence of various infections in Iranian SLE patients and its contribution to mortality during a 21 years period (1974-97).

Methods We evaluated retrospectively 1165 patients for 21 years. For each patient 266 parameters were systemically checked. Each episode of infection was evaluated according to the organic involvement, type of infection and the infective agent, presence of steroid therapy.

Results During this 21 years period 393 infection episodes were noted in 224 patients. The total follow-up time was 820 years (mean: 3.66). The mean interval between the beginning of the illness and the occurrence of the infectious episode was 3.4 years. Urinary tract infection (UTI) was the most frequent (24.9\%). The UTI was seen in $9.3 \%$ of patients. The most frequent bacteria was Escherichia coli (68.4\% of UTI). Pulmonary infections were seen in $11.9 \%$ of infection cases. In infectious pneumonia, $72.2 \%$ was due to Pneumococci. Septic arthritis was seen in 17 cases (4.3\% of all infections) and Osteomyelitis in 5 cases $(1.3 \%)$. The most frequent bacteria for musculoskeletal infections were Salmonella. Soft tissue infections (abscesses, cellulitis, infectious ulcer and infectious lymphadenitis) were seen in 67 cases $(17 \%)$. The most frequent bacteria were Staphylococcus aureus. Septicemia was seen in 64 cases (16.3\%) of patients. The most frequent bacteria were salmonella. The mean steroid dose in patients who died of infection was $48.6 \mathrm{mg} /$ day versus $25.3 \mathrm{mg}$ in others $(\mathrm{P}<0.0001)$. To determine the effectivity of simple laboratory tests in early diagnosis of severe infections, patients were divided into three groups according to the severity of their illness. Severe infections were accompanied with higher levels of ESR ( $P=0.0005)$, CRP $(P=0.00001)$ and serum creatinine $(\mathrm{P}=0.00001)$ and lower levels of serum complement $(\mathrm{P}$ $=0.006$ ) while Anti-DNA and active urinary sediment were not among differentiating factors. There was a significant increase in WBC count in severe infections when steroid dose was below 40 $\mathrm{mg} /$ day $(\mathrm{P}<0.0001$ versus $\mathrm{P}<0.4$ when the steroid dose was more than $40 \mathrm{mg}$ ).

Conclusion UTI was the most common infection and E. coli was the most frequent infective organism. Apart lower UTI, soft tissue infections, sepsis, and pulmonary infections were the commonest infections while $S$. aureus and salmonella were the most common agents. Moreover, high dose of steroids is a significant predisposing factor for infections as mentioned in other published studies. A high WBC count, raised CRP and ESR can be helpful for the diagnosis of an infection with organisms such as Salmonella, Staphylococcus, Pseudomonas or Candida albicans.

\section{OP0103 SYSTEMIC LUPUS ERYTHEMATOSUS IS ASSOCIATED WITH MORE MORTALITY AND TISSUE DAMAGE IN MEN THAN IN WOMEN}

FJ López-Longo, CM González, M Moreno-Zazo, CA Montilla, R Del Castillo, N Caro, L Cebrián, C González-Montagut, S Gómez, E Carmona, I Monteagudo, L Carreño. Rheumatologhy, Hospital General Universitario Gregorio Marañón, Madrid, Spain

\subsection{6/annrheumdis-2001.187}

Background Systemic lupus erythematosus (SLE) is less frequent in men than in women, but it can be more severe.

Objectives To determine if the outcome of SLE and its treatment differ between men and women. 